\title{
NEURAL NETWORK BASED IDENTIFICATION OF HYSTERESIS IN HUMAN MERIDIAN SYSTEMS
}

\author{
Yonghong TAN*, Ruili DONG* ${ }^{*}$, Hui CHEN ${ }^{* *}$, Hong HE* \\ * College of Mechanical and Electronic Technology \\ Shanghai Normal University, \#100 Guilin Road, Shanghai, 200234, China \\ e-mail: \{dongrlnpu, tany\} ashnu. edu.cn \\ ${ }^{* *}$ Department of Automation \\ Southeast University, \#2 Si-Pai-Lou Street, Nanjing, 210096, Nanjing, China
}

\begin{abstract}
Developing a model based digital human meridian system is one of the interesting ways of understanding and improving acupuncture treatment, safety analysis for acupuncture operation, doctor training, or treatment scheme evaluation. In accomplishing this task, how to construct a proper model to describe the behavior of human meridian systems is one of the very important issues. From experiments, it has been found that the hysteresis phenomenon occurs in the relations between stimulation input and the corresponding response of meridian systems. Therefore, the modeling of hysteresis in a human meridian system is an unavoidable task for the construction of model based digital human meridian systems. As hysteresis is a nonsmooth, nonlinear and dynamic system with a multi-valued mapping, the conventional identification method is difficult to be employed to model its behavior directly. In this paper, a neural network based identification method of hysteresis occurring in human meridian systems is presented. In this modeling scheme, an expanded input space is constructed to transform the multi-valued mapping of hysteresis into a one-to-one mapping. For this purpose, a modified hysteretic operator is proposed to handle the extremum-missing problem. Then, based on the constructed expanded input space with the modified hysteretic operator, the so-called Extreme Learning Machine (ELM) neural network is utilized to model hysteresis inherent in human meridian systems. As hysteresis in meridian system is a dynamic system, a dynamic ELM neural network is developed. In the proposed dynamic ELM neural network, the output state of each hidden neuron is fed back to its own input to describe the dynamic behavior of hysteresis. The training of the recurrent ELM neural network is based on the least-squares algorithm with $\mathrm{QR}$ decomposition.
\end{abstract}

Keywords: acupuncture point, hysteresis, identification, neural networks, human meridian, traditional Chinese medicine.

\section{Introduction}

Acupuncture and meridian studies have a history of more than three thousand years in China. They are one of the most important parts of traditional Chinese medicine. Based on the theory of traditional Chinese medicine, it has been found that acupuncture points are distributed in the meridian system of the human body. Moreover, the meridian system is an independent system which exists in the body parallel with neural systems and blood circulation systems (Tsuei, 1998; Trentini and Erlichman, 2005). Experimental results have shown that the meridian system has significant effect on human health (Tsuei, 1998). Based on the recent research results, it has been illustrated that the meridian system has architecture with many channels allowing the electrical signals to pass through easily
(Zhang and Zhu, 1999; Yang, 1997).

A review of electrical properties of acupuncture points and meridians has been provided by Ahn and Langevin (2008). That could be used to explain why acupuncture therapy would treat some diseases in the human body by implementing some electrical stimulation signals on the related acupuncture points. The acupuncture points distributed in the meridian system offer some distinctive ways of transferring signals and processing information including electrical information (Yang, 1997).

Until today, there have been some research results on human meridian system focusing on the analysis of impedance on a single acupuncture point (Yamamoto and Yamamoto, 1979; Yang, 1997; Zhang and Zhu, 1999). On the other hand, a numerical study has been performed on bio-fluid dynamics to explore and provide some human 
meridian characteristics (Ahn and Langevin, 2008). However, the human meridian system is a network with several channels. In each channel, there are several acupuncture points located along a curve. Experimental results demonstrated that there were some relations among those points in each channel. Therefore, the analysis depending just on the impedance of one single acupuncture point would not reflect the main characteristic of the signal transmission in the human meridian system. One of the options is to use an excitation signal to stimulate an acupuncture point in a channel of the meridian. Then the corresponding responses of the other acupuncture points in the same channel are measured. The signal transmission performance of the measured channel in the meridian can be evaluated.

There are many reasons for developing proper models of human meridian systems. For example, models that accurately describe the behavior of human meridian systems could be used for understanding and improving the acupuncture treatment, safety analysis for acupuncture operation, doctor training, or treatment scheme evaluation. Moreover, the so-called digital human meridian system could be constructed by a number of models to describe human meridian behavior.

The experimental results revealed that the human meridian system is a dynamic one (Zhang and Zhu, 1999; Yang, 1997; Wang and Su, 2009). In this case, the identification of the model to describe the dynamic behavior of the meridian is an efficient method for performance evaluation, simulation, and design of optimal stimulation for disease treatment. Wang and $\mathrm{Su}$ (2009) developed an auto-regressive and moving average model to describe the human meridian system. It fits the response well when the exciting signal is of low frequency and the input amplitude is rather small. However, when the frequency of the exciting input is higher or the amplitude of the exciting signal is larger, it exhibits some nonlinear behavior, especially hysteretic. Thus, a nonlinear dynamic model should be considered to describe this system.

In this paper, the experimental configuration to measure the responses of the acupuncture points of the meridian stimulated by electric signals is presented. Then, the phenomenon of the obtained experimental results is analyzed. It is found out that as the frequency of the stimulation signal increases, the phenomenon of hysteresis occurs in the meridian. It is known that the model of hysteresis is a challenge due to non-smoothness, memory and multivalued mapping of hysteresis.

In our previous work (Zhao, 2006; Zhao and Tan, 2008), the so-called expanded input space method has been proposed. In this method, a hysteretic operator which extracts the moving tendency of hysteresis is introduced as another coordinator of the input space. Then, based on the input and the output of the hysteretic operator, the output of hysteresis can be uniquely determined. Thus, the multi-valued mapping of hysteresis can be trans- formed to a one-to-one mapping. However, the extrememissing problem may be met in the hysteretic operator. It may lead to a larger modeling error.

In this paper, we extend the idea of the hysteretic operator to handle the extreme-missing problem of the operator. Then a modified hysteretic operator is proposed. In order to transform the multi-valued mapping of hysteresis to a one-to-one mapping, an expanded input space is constructed with the introduced modified hysteretic operator. Hence, based on the constructed expanded input space, a Dynamic Extreme Learning Machine (DELM) neural network is applied to the identification of the hysteretic characteristic occurring in the human meridian. One of the most important advantages of the DELM is that it can avoid the local minima in the optimization because the training of the neural network becomes a convex problem. After that, the corresponding experimental results of modeling are presented. In the experiment, both the traditional ELM neural network and the proposed DELM version are implemented to model hysteresis in the human meridian for comparison. Moreover, a backpropagation neural network based on the expanded input space method is also utilized to model the hysteresis for comparison.

\section{Experimental configuration and brief analysis}

In order to investigate the behavior of human the meridian in electrical stimulation, in this section, a method based on three detecting electrodes is used to measure both stimulation and the corresponding response of the acupuncture points in meridian systems. The configuration of the measurement for the meridian response is shown in Fig. 1. Based on the theory of Chinese medicine, there are 11 acupoints in the so-called Hand-Taiyin Lung Channel.

In this experiment, the stimulating acupoint was named Shaoshang (LU 11), the acupoint Tianfu (LU 3) was connected to the ground of the circuit, and the detecting acupoints were Chize (LU 5), Kongzui (LU 6) and Lieque (LU 7). The stimulation voltage signals were generated by a signal generator.

In this experiment, the acupoint Shaoshang (LU11) was excited by the stimulation signals through a stimulating electrode. Then, the detecting electrodes were used to measure the corresponding responses of the acupoints Chize (LU5) and Kongzui (LU6) simultaneously. The measured output was sampled by an Analog/Digital (A/D) converter. The sampled signals were sent to the computer for further processing. Before the test, volunteers were relaxed to avoid the strenuous disturbance. In the experiments, a very interesting thing is that hysteresis phenomenon occurs in human meridian systems when we stimulate the acupoints in the meridian using a sequence of sine wave samples with the frequency of $10 \mathrm{KHz}$. Figure 2 shows the plot of the output of the acupoint LU5 


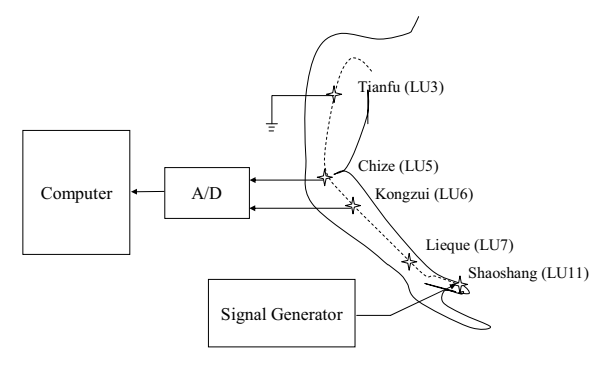

Fig. 1. Experimental configuration of meridian signal measurement.
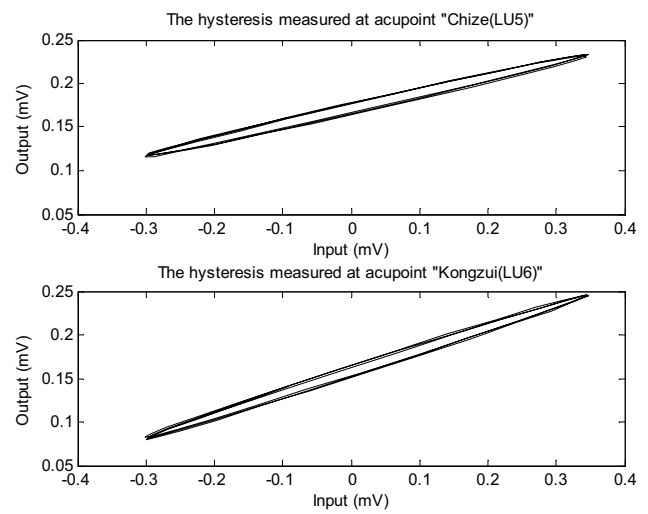

Fig. 2. Hysteretic phenomena occurring in the human meridian (frequency of input: $10 \mathrm{kHz}$ ).
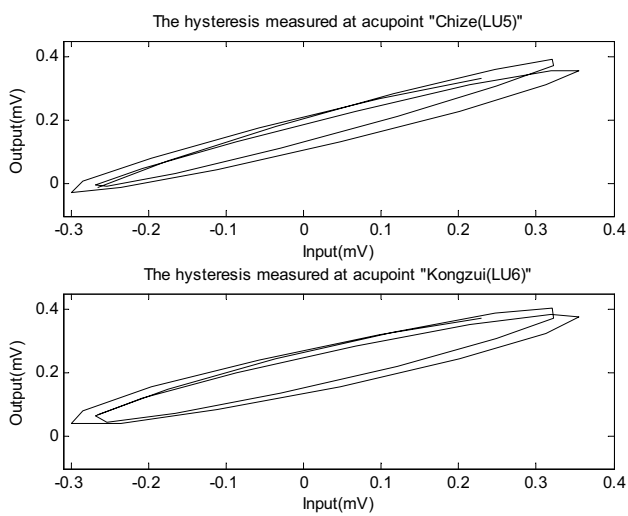

Fig. 3. Hysteretic phenomena occurring in the human meridian (frequency of input: $100 \mathrm{kHz}$ ).

versus the input as well as the curve of the output of the acupoint LU6 against the input.

When the frequency of the input increases to
$100 \mathrm{KHz}$, the corresponding hysteresis also happened at the above-mentioned meridian points, shown in Fig. 3. It can be seen that the hysteresis occurring in the meridian changed its performance as the input frequency increases. The width of the hysteresis increases and the hysteresis rotates to an angle in the clockwise direction as the input frequency increases. Obviously, the hysteresis presents dynamic behavior relying on the input frequency.

Moreover, we can see that the response of LU6 is much different from that of LU5 for the same exciting input, especially when the input frequency increases to 10 $\mathrm{KHz}$. The slope values of the curve for LU6 are larger than those for LU5. As the acupoint LU6 is closer to the exciting point LU11, clearly, the gain of this acupoint is larger than that of the acupoint LU5. It implies that the effect of the stimulation will be reduced as the distance between the detected acupoint and the exciting acupoint increases. From Figs. 2 and 3, we note that the hysteretic phenomenon occurred in human meridian systems with at least the following characteristics:

(i) non-smoothness,

(ii) multi-valued mapping.

The hysteretic behaviour in human meridian response might be explained as tension and spasm caused by external stimulation. It is clear that it is a dynamic system with rather complex characteristics. As the conventional identification methods can only be useful for systems with smooth and one-to-one mappings, it is a challenge to construct a model of hysteresis.

\section{Expanded input space with a modified hysteretic operator}

It has been mentioned that hysteresis is a non-smooth system with multi-valued mapping. Usually, conventional methods of system identification are only available for systems with one-to-one mapping. Moreover, as hysteresis is a nonlinear system, the optimization technique is usually used in the identification of nonlinear systems. In this case, conventional optimization methods might be fail in the identification of hysteresis since the gradients do not exist at non-smooth points of hysteresis.

Up to now, there have been some methods of hysteresis modeling, e.g., the Preisach model (Hu and Mrad, 2003) and the Prandtl-Ishlinskii (PI) model (Macki, 1993). Those methods used simple backlash operators as the basis functions for modeling. Therefore, lots of operators should be employed in order to obtain accurate models. Although there have been some modified Preisach models (Hu and Mrad, 2003; Ge and Jouaneh, 1995) as well as a modified PI model (Dong and Tan, 2009) proposed to describe hysteresis systems, the structures of those modified schemes are still very complex. In order 
to simplify the model architecture to describe the behavior of hysteresis, Li and Tan (2004) as well as Zhao and Tan (2006) developed the so-called expanded input space based hysteretic models.

In the expanded input space, a hysteretic operator which extracted the main movement feature of hysteresis was introduced as one of the coordinates. Thus, the multi-valued mapping of hysteresis can be transformed to a one-to-one mapping between the inputs and the output. Then, feedforward neural networks were implemented to model hysteresis based on the constructed expanded input space. An advantage of the method of the expanded input space is that it can result in a model with a simple structure, avoiding the computation of gradients.

In this section, based on our previous work (Zhao and Tan, 2006; 2008), a modified hysteretic operator is proposed. The modified hysteretic operator will handle the extreme-missing problem. Then, we construct an expanded input space with a modified hysteretic operator, which is a two-dimensional plane consisting of the input as well as the output of the hysteretic operator. As the hysteretic operator can extract the movement characteristics, such as an increase, a decrease and a turning of hysteresis, on this plane, the output of the hysteresis corresponding to the point in the input plane can be uniquely determined. One of the advantages of the expanded input space is that the one-to-one mapping between the input space and the output of the hysteresis can be constructed. Thus, techniques for nonlinear modeling can be implemented to model the behavior of hysteresis on the constructed input space. Moreover, another advantage of this modeling method is that the computation of gradients at non-smooth points can be avoided.

Assumption 1. Suppose the hysteresis is continuous and forms a closed loop in the input-output diagram when the input cycles between two extrema. Then, we define the hysteresis operator $f(x)$ as

$$
f(x)=\left(1-e^{\left|\left(x-x_{p}\right)\right|}\right)+f\left(x_{p}\right) .
$$

where $x$ is the current input, $f(x)$ is the current output, $x_{p}$ is the dominant extremum adjacent to the current input $x$, $f(x)$ is the output of the operator when the input is $x$.

The properties of the hysteretic operator are as follows:

(i) Let $x(t) \in C\left(\mathbb{R}^{+}\right)$, where $\mathbb{R}^{+}=(t \mid t \geq 0)$, and let $C\left(\mathbb{R}^{+}\right)$be the set of continuous functions on $\mathbb{R}^{+}$. For the different time instants $t_{1}$ and $t_{2}$, we have $t_{1} \neq t_{2}$ but $x\left(t_{1}\right)=x\left(t_{2}\right)$, where $x\left(t_{1}\right)$ and $x\left(t_{2}\right)$ are not the extrema. Then it leads to $f\left[x\left(t_{1}\right)\right] \neq f\left[x\left(t_{2}\right)\right]$.

(ii) If there exist two time instants $t_{1}$ and $t_{2}, t_{1} \neq t_{2}$, such that $f\left[x\left(t_{1}\right)\right]-f\left[x\left(t_{2}\right)\right] \rightarrow 0$, then $x\left(t_{1}\right)=$ $x\left(t_{2}\right) \rightarrow 0$. Therefore, Lemmas 1 and 2 given by
Zhao (2006) are presented in the following, respectively.

Lemma 1. Let $x(t) \in C\left(\mathbb{R}^{+}\right)$. If there exist two time instants $t_{1}$ and $t_{2}, t_{1} \neq t_{2}$, such that $x\left(t_{1}\right)=x\left(t_{2}\right), x\left(t_{1}\right)$ and $x\left(t_{2}\right)$ are not the extrema, then $f\left[x\left(t_{1}\right)\right] \neq f\left[x\left(t_{2}\right)\right]$.

Lemma 2. If there exist two time instants $t_{1}$ and $t_{2}$, $t_{1} \neq t_{2}$, such that $f\left[x\left(t_{1}\right)\right]-f\left[x\left(t_{2}\right)\right] \rightarrow 0$, then $x\left(t_{1}\right)-$ $x\left(t_{2}\right) \rightarrow 0$

Remark 1. Note that Lemma 1 does not consider the case when there is no output extremum of the hysteretic operator as the input just passes through the input extremum. For example, suppose that $t_{1}$ and $t_{2}\left(t_{1}<t_{2}\right)$ are two time instants. In this case, if the corresponding input values at those two time instants are equal to each other, but the output of the hysteretic operator corresponding to one of the input value is in the increase zone while the output of the operator with respect to another input value is in the decrease zone. The extrema between those two output values of the hysteresis are obviously missing.

To handle the extremum-missing problem, a modified scheme of the hysteresis operator is proposed as follows.

Theorem 1. For the formula (1), if $x\left(t_{1}\right)=x\left(t_{2}\right)$, $f\left[x\left(t_{1}\right)\right] \neq f\left[x\left(t_{2}\right)\right]$, where $x\left(t_{1}\right)$ and $x\left(t_{2}\right)$ are the adjacent time instants and $t_{1}<t_{2}$, an extremum located in the segment between points $\left(x\left(t_{1}\right), f\left(x\left(t_{1}\right)\right)\right)$ and $\left(x\left(t_{2}\right), f\left(x\left(t_{2}\right)\right)\right)$ cannot be obtained within the time period $\left[t_{1}, t_{2}\right]$. However, it can be estimated by $\left(x_{m}, f\left(x_{m}\right)\right)$, where

$$
x_{m}=x\left(t_{2}\right)+0.5\left(x\left(t_{2}\right)-x\left(t_{1}\right)\right),
$$

and

$$
\begin{aligned}
& f\left(x_{m}\right) \\
& =\left\{\begin{array}{r}
\left(1-e^{\left(-x_{m}+x_{p}\right)}\right)\left(x_{m}-x_{p}\right)+f\left(x_{p}\right) \\
\text { if } x\left(t_{2}\right)>x\left(t_{1}\right), \\
\left(1-e^{\left(x_{m}-x_{p}\right)}\right)\left(x_{m}-x_{p}\right)+f\left(x_{p}\right) \\
\text { if } x\left(t_{2}\right)<x\left(t_{1}\right) .
\end{array}\right.
\end{aligned}
$$

Proof. Suppose that $x_{p}$ is the local maximum of the input, whilst $x\left(t_{1}\right)$ and $x\left(t_{2}\right)$ are located in the increase and decrease zones, respectively. Hence, the derivatives of $f(x(t))$ with $x(t)$ respect to $x\left(t_{1}\right)$ and $x\left(t_{2}\right)$ are

$$
\dot{f}\left(x\left(t_{2}\right)\right)=e^{\left(-x\left(t_{2}\right)+x_{p}\right)}
$$

and

$$
\dot{f}\left(x\left(t_{1}\right)\right)=e^{\left(-x\left(t_{1}\right)+x_{p}\right)},
$$

respectively.

Based on the assumption given by Zhao (2006), $\left(x_{p}, f\left(x_{p}\right)\right)$ is a local minimum, and $\left(x\left(t_{1}\right), f\left(x\left(t_{1}\right)\right)\right)$ is a 
point adjacent to the local maximum point $\left(x_{m}, f\left(x_{m}\right)\right)$. Hence, $\dot{f}\left(x\left(t_{2}\right)\right) \approx \dot{f}\left(x\left(t_{1}\right)\right)$ due to the properties of the exponential function. That is to say, the three points, i.e., $\left(x\left(t_{1}\right), f\left(x\left(t_{1}\right)\right)\right),\left(x\left(t_{2}\right), f\left(x\left(t_{2}\right)\right)\right)$ and $\left(x_{m}, f\left(x_{m}\right)\right)$, can be considered to be approximately located on a straight line. Moreover, $\left(x_{m}, f\left(x_{m}\right)\right)$ is the point between $\left(x\left(t_{1}\right), f\left(x\left(t_{1}\right)\right)\right)$ and $\left(x\left(t_{2}\right), f\left(x\left(t_{2}\right)\right)\right)$, where $t_{1}$ and $t_{2}$ are the adjacent time instants. Define $L\left(t_{2}, t_{1}\right)$ that represents the curve connecting $\left(x\left(t_{1}\right), f\left(x\left(t_{1}\right)\right)\right)$ with $\left(x\left(t_{2}\right), f\left(x\left(t_{2}\right)\right)\right)$. So the extremum point in the space can be approximated by the mean values of the projections of $L\left(t_{2}, t_{1}\right)$ on each coordinate in the plane. In other words, the mean value of the projection on the input coordinate is estimated by

$$
x_{m}=x\left(t_{2}\right)+0.5\left(x\left(t_{2}\right)-x\left(t_{1}\right)\right),
$$

while the mean value of the projection on the coordinate of the output of the hysteretic operator is estimated by

$$
f\left(x_{m}\right)=\left(1-e^{\left(-x_{m}+x_{p}\right)}\right)\left(x_{m}-x_{p}\right)+f\left(x_{p}\right) .
$$

Similarly, if $\left(x_{m}, f\left(x_{m}\right)\right)$ is a local minimum, $f\left(x_{m}\right)$ can be described by

$$
f\left(x_{m}\right)=\left(1-e^{\left.\left(x_{m}-x_{p}\right)\right)}\left(x_{m}-x_{p}\right)+f\left(x_{p}\right) .\right.
$$

Hence, combining Eqns. (6)-(8) leads to

$$
\begin{aligned}
& f\left(x_{m}\right) \\
& =\left\{\begin{array}{l}
\left(1-e^{\left(-x_{m}+x_{p}\right)}\right)\left(x_{m}-x_{p}\right)+f\left(x_{p}\right), \\
x\left(t_{2}\right)>x\left(t_{1}\right), \\
\left(1-e^{\left(x_{m}-x_{p}\right)}\right)\left(x_{m}-x_{p}\right)+f\left(x_{p}\right), \\
x\left(t_{2}\right)<x\left(t_{1}\right) .
\end{array}\right.
\end{aligned}
$$

Theorem 2. For any hysteresis satisfying Assumption 1, there exists a continuous one-to-one mapping $\Gamma: \mathbb{R}_{2} \rightarrow$ $\mathbb{R}$, such that $H[x(t)]=\Gamma[x(t), f(x(t))]$.

Proof. Firstly, let us prove that $\Gamma$ is a one-to-one mapping. Consider the following cases.

Case 1. Assume that $x(t)$ is not an extremum. In terms of Lemma 1 , if there exist two different time instances $t_{1}$ and $t_{2}$, then $\left(x\left(t_{1}\right), f\left[x\left(t_{1}\right)\right]\right) \neq\left(x\left(t_{2}\right), f\left[x\left(t_{2}\right)\right]\right)$. Therefore, the coordinate $(x(t), f[x(t)])$ uniquely corresponds to the hysteresis $H[x(t)]$.

Case 2. Suppose that $x(t)$ is an extremum. In this case, for two different time instances $t_{1}$ and $t_{2}$, there will be $\left(x\left(t_{1}\right), f\left[x\left(t_{1}\right)\right]\right)=\left(x\left(t_{2}\right), f\left[x\left(t_{2}\right)\right]\right)$.

Case 3. Suppose that $\left.x\left(t_{1}\right)=x\left(x_{2}\right), f\left[x\left(t_{1}\right)\right]\right) \neq$ $f\left[x\left(t_{2}\right)\right]$, where $t_{1}$ and $t_{2}$ are the adjacent time instants and $t_{1}<t_{2}$, while the extrema located in the segment between points $\left(x\left(t_{1}\right), f\left(x\left(t_{1}\right)\right)\right)$ and $\left(x\left(t_{2}\right), f\left(x\left(t_{2}\right)\right)\right)$ cannot be obtained within the time period $\left[t_{1}, t_{2}\right]$. In this situation, based on Theorem 1, we can use the mean values between those two points, i.e., $\left(x_{m}, f_{m}\left(t_{2}, t_{1}\right)\right)$ to approximate the extrema. According to the property of the Preisach-type hysteresis, $H\left[x\left(t_{1}\right)\right]=H\left[x\left(t_{2}\right)\right]$. Then the coordinate $(x(t), f[x(t)])$ will be uniquely corresponding to the hysteresis $H[x(t)]$.

Combining the above-mentioned three situations, we obtain that $\Gamma$ is a one-to-one mapping. Next, we will verify that $\Gamma$ is a continuous mapping.

According to the property of the Preisach-type hysteresis, if

$$
x\left(t_{1}\right)-x\left(t_{2}\right) \rightarrow 0,
$$

it yields

$$
H\left[x\left(t_{1}\right)\right]-H\left[x\left(t_{2}\right)\right] \rightarrow 0 .
$$

Then, considering Lemma 2, if

$$
f\left[x\left(t_{1}\right)\right]-f\left[x\left(t_{2}\right)\right] \rightarrow 0,
$$

we can deduce that

$$
x\left(t_{1}\right)-x\left(t_{2}\right) \rightarrow 0 .
$$

Then we have $H\left[x\left(t_{1}\right)\right]-H\left[x\left(t_{2}\right)\right] \rightarrow 0$. Therefore, it can be concluded that there exists a continuous oneto-one mapping $\Gamma: \mathbb{R}^{2} \rightarrow \mathbb{R}$, such that $H[x(t)]=$ $\Gamma[x(t), f(x(t))]$.

Remark 2. The theorem stated above indicates that the constructed expanded input space consists of two coordinates, i.e., $x$ and $f(x)$. Figure 4 demonstrates how a one-to-one mapping can be derived on the expanded input space. From Fig. 4, it is illustrated how the multi-valued mapping of the hysteresis is transformed into a one-toone mapping on this constructed input space. Suppose that $x=x_{u}$. Then, it yields the corresponding outputs of the hysteretic operator, i.e., $f_{a}\left(x_{u}\right)$ and $f_{b}\left(x_{u}\right)$, respectively. Then, we have two points on the constructed expanded input space, i.e., $a\left(x_{u}, f_{a}\left(x_{u}\right)\right)$ and $b\left(x_{u}, f_{b}\left(x_{u}\right)\right)$, respectively. In this case, the corresponding output values of the hysteresis, i.e., $A=\Gamma\left(x_{u}, f_{a}\left(x_{u}\right)\right)$ and $B=$ $\Gamma\left(x_{u}, f_{b}\left(x_{u}\right)\right)$, can be uniquely specified.

Remark 3. Based on Theorem 2, the modified hysteretic operator is a combination of Eqns. (1)-(3). We can use the modified hysteretic operator to construct an expanded input space.

It has also been proved that the expanded input space is a compact set (Zhao and Tan, 2006). Hence, the mapping between the output and the input of hysteresis on this expanded input space is a one-to-one mapping. Thus, the neural networks such as the multilayer feedforward neural network can be implemented to model the performance of hysteresis on this input space. 


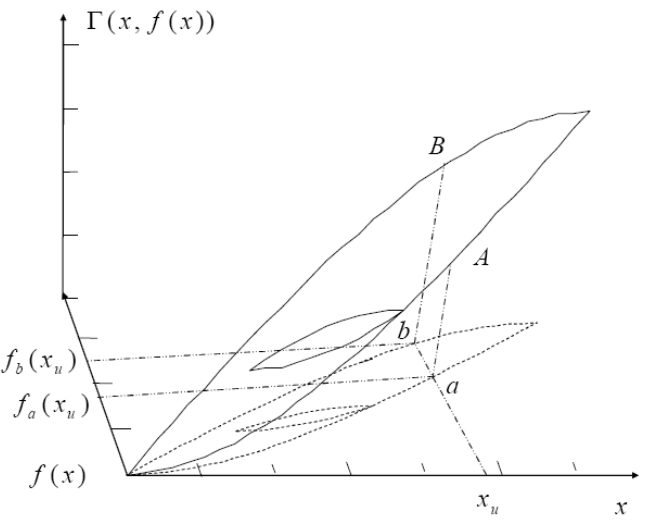

Fig. 4. Transformation on the expanded input space.

However, due to the non-convex characteristics of feedforward neural networks, one often meets the problem that the training gets easily stuck in local minima. It would have significant effect on the performance of the obtained neural models.

\section{Dynamic ELM neural model of hysteresis in meridian systems}

In this section, a dynamic extreme learning machine neural model is proposed to model hysteresis in meridian systems based on the expanded input space proposed above. Recently, Huang and Siew (2006) as well as Huang and Chen (2007) proposed the so-called extreme learning machine neural network with randomly specified input weights of the single hidden layer and the output weights of the network to be determined by using the least squares algorithm. ELM has achieved very good performance in generalization along with much faster learning speed. It may avoid the drawbacks of feedforward neural networks, which often get stuck in some local minima during the training procedure. A brief description of the ELM neural network is presented as follows.

For $N$ data samples $\left\{\left(x_{k}, t_{k}\right)\right\}_{k=1}^{N}$, where $\mathbf{x}_{k}=$ $\left[x_{k 1}, x_{k 2}, \ldots, x_{k n}\right]$ is the $k$-th input vector and $\mathbf{t}_{k}=$ $\left[t_{k 1}, t_{k 2}, \ldots, t_{k n}\right]$ is the $k$-th target vector, a Single Layer Feedforward Network (SLFN) has $\tilde{N}$ hidden neurons and an activation function, which is a sigmoid function i.e.,

$$
\sum_{i=1}^{\tilde{N}} \boldsymbol{\beta}_{i} g\left(\mathbf{w}_{i} \cdot \mathbf{x}_{k}+b_{i}\right)=\mathbf{o}_{k}, \quad k=1, \ldots, N
$$

where $\mathbf{w}_{i}=\left[w_{i 1}, w_{i 2}, \ldots, w_{i n}\right]^{T}$ is the weight vector connecting the $i$-th hidden neuron and the input neurons, $\boldsymbol{\beta}_{i}=\left[\beta_{i 1}, \beta_{i 2}, \ldots, \beta_{i n}\right]^{T}$ is the weight vector connecting the $i$-th hidden neuron and output neurons, $\mathbf{o}_{k}=$ $\left[o_{k 1}, o_{k 2}, \ldots, o_{k n}\right]^{T}$ is the output vector of the SLFN, and $b_{i}$ is the threshold of the $i$-th hidden neuron. Moreover, $\mathbf{w}_{i} \cdot \mathbf{x}_{k}$ denotes the inner product of $\mathbf{w}_{i}$ and $\mathbf{x}_{k}$. Hence, these $N$ equations can be written compactly as

$$
\mathbf{H} \boldsymbol{\beta}=\mathrm{O},
$$

where $\mathbf{H}$ is the hidden layer output matrix, i.e.,

H

$$
=\left(\begin{array}{ccc}
g\left(\mathbf{w}_{1} \cdot \mathbf{x}_{1}+b_{1}\right) & \ldots & g\left(\mathbf{w}_{\tilde{N}} \cdot \mathbf{x}_{1}+b_{\tilde{N}}\right) \\
\vdots & \ldots & \vdots \\
g\left(\mathbf{w}_{1} \cdot \mathbf{x}_{N}+b_{1}\right) & \ldots & g\left(\mathbf{w}_{\tilde{N}} \cdot \mathbf{x}_{N}+b_{\tilde{N}}\right)
\end{array}\right)_{N \times \tilde{N}},
$$

$$
\boldsymbol{\beta}=\left(\begin{array}{c}
\boldsymbol{\beta}_{1}^{T} \\
\vdots \\
\boldsymbol{\beta}_{\tilde{N}}^{T}
\end{array}\right)_{\tilde{N} \times m}
$$

and

$$
\mathbf{O}=\left(\begin{array}{c}
\mathbf{o}_{1}^{T} \\
\vdots \\
\mathbf{o}_{\tilde{N}}^{T}
\end{array}\right)_{N \times m}
$$

By randomly assigning and fixing the input weights $\mathbf{w}_{i}$ and biases $b_{i}$, only output weights $\boldsymbol{\beta}_{i}$ are left to be tuned by minimizing the following objective function:

$$
J=\|\mathbf{H} \boldsymbol{\beta}-\mathbf{T}\|_{2}^{2} .
$$

According to Theorem 2 in Section 3, hysteresis existing in meridian systems can be identified using the ELM neural network on the constructed expanded input space. Hence, the corresponding ELM neural model for hysteresis is shown as follows:

$$
\begin{aligned}
& \boldsymbol{\Gamma}[x, f(x)] \\
& =\mathbf{H}[x, f(x)] \boldsymbol{\beta}+\varepsilon \\
& =\left(\begin{array}{c}
g\left(\mathbf{w}_{1} \cdot\left[\mathbf{x}_{1}, f\left(\mathbf{x}_{1}\right)\right]+b_{1}\right) \\
\vdots \\
g\left(\mathbf{w}_{1} \cdot\left[\mathbf{x}_{N}, f\left(\mathbf{x}_{N}\right)\right]+b_{1}\right) \\
\quad \ldots \\
\quad g\left(\mathbf{w}_{\tilde{N}} \cdot\left[\mathbf{x}_{1}, f\left(\mathbf{x}_{1}\right)\right]+b_{\tilde{N}}\right) \\
\vdots \\
\left.\mathbf{w}_{\tilde{N}} \cdot\left[\mathbf{x}_{N}, f\left(\mathbf{x}_{N}\right)\right]+b_{\tilde{N}}\right)
\end{array}\right)\left(\begin{array}{c}
\boldsymbol{\beta}_{1}^{T} \\
\vdots \\
\boldsymbol{\beta}_{\tilde{N}}^{T}
\end{array}\right)+\varepsilon
\end{aligned}
$$

where $\varepsilon$ is the modeling error vector, for any given $\varepsilon_{N}>$ $0,\|\varepsilon\| \leq \varepsilon_{N}$.

As hysteresis in the human meridian system is a dynamic system, to describe the dynamic behavior of hysteresis in the meridian system, an internal feedback connection is introduced for each hidden neuron in the ELM neural network to memorize the historic states of the network. Thus, a dynamic ELM neural network on the expanded input space is constructed for hysteresis inherent in the human meridian. Hence, we have

$$
\begin{aligned}
& \boldsymbol{\Gamma}[\mathbf{x}(t), f(\mathbf{x}(t))] \\
& \quad=\mathbf{H}[\mathbf{x}(t), f(\mathbf{x}(t)), z(\mathbf{x}(t-1), t-1)] \boldsymbol{\beta}]+\varepsilon,
\end{aligned}
$$


where

$$
\boldsymbol{\beta}=\left(\begin{array}{c}
\boldsymbol{\beta}_{1}^{T} \\
\vdots \\
\boldsymbol{\beta}_{\tilde{N}}^{T}
\end{array}\right)
$$

and $\mathbf{H}(\mathbf{x}(t), f(\mathbf{x}(t)), z(\mathbf{x}(t-1), t-1)]$ is given by (18) where $\alpha_{i}$ is the feedback factor with the value randomly assigned within $(0,1)$ to guarantee the model stability.

Theorem 3. Suppose that the active function of the neuron $g(\cdot)$ is a sigmoid function. If the assigned value of the feedback factor $\alpha_{i}$ is constrained to $(0,1)$, then the output of each neuron, i.e.,

$$
\begin{aligned}
& z_{i}\left(\mathbf{x}_{j}(t), t\right) \\
& =g\left(\mathbf{w}_{i} \cdot\left[\mathbf{x}_{j}(t), f\left(\mathbf{x}_{j}(t)\right)\right]\right) \\
& +\alpha_{i} z_{i}\left(\mathbf{x}_{j}(t-1), t-1\right), \\
& \quad i=1, \ldots, N, \quad j=1, \ldots, \tilde{N} .
\end{aligned}
$$

will satisfy $\left|z_{i}\left(\mathbf{x}_{j}(t), t\right)\right| \leq 1, i=1, \ldots, N$, as $t \rightarrow \infty$.

Proof. Considering $g(\cdot)$ as a sigmoid function leads to $|g(\cdot)| \leqslant 1$. For the $i$-th neuron shown in (19), it can be described by

$$
z_{i}\left(\mathbf{x}_{j}(t), t\right)=\frac{g\left(\mathbf{w}_{i}\left[\mathbf{x}_{i}(t), f\left(\mathbf{x}_{i}(t)\right)\right]\right)}{\left(1-\alpha_{i} q^{-1}\right)}
$$

where $q^{-1}$ is the unit back shift operator. If $0<\alpha_{i}<1$,

$$
z_{i}\left(\mathbf{x}_{j}(t), t\right)=\frac{g\left(\mathbf{w}_{i}\left[\mathbf{x}_{i}(t), f\left(\mathbf{x}_{i}(t)\right)\right]\right)}{\left(1-\alpha_{i} q^{-1}\right)}
$$

will be asymptotically stable. Thus, there exists $0<\zeta_{i} \leq$ 1 such that $\lim _{t \rightarrow \infty}\left|z_{i}\left(\mathbf{x}_{j}(t), t\right)\right|<\zeta_{i} \leqslant 1$.

Just as has been mentioned above, all the input weights $\mathbf{w}_{i}$ and biases $b_{i}$ of the neurons are fixed with the assigned random values, and all the feedback factors $\alpha_{i}$ are fixed with random values assigned within $(0,1)$. In this case, we have

$$
\boldsymbol{\Gamma}[\mathbf{x}(t), f(\mathbf{x}(t)]=\mathbf{Z}(\mathbf{x}(t), t) \boldsymbol{\beta}+\varepsilon,
$$

where

$$
\mathbf{Z}(\mathbf{x}(t), t)=\left(\begin{array}{ccc}
z_{1}\left(\mathbf{x}_{1}(t), t\right) & \ldots & z_{\tilde{N}}\left(\mathbf{x}_{1}(t), t\right) \\
\vdots & \ldots & \vdots \\
z_{1}\left(\mathbf{x}_{N}(t), t\right) & \ldots & z_{\tilde{N}}\left(\mathbf{x}_{N}(t), t\right)
\end{array}\right)
$$

and only the unknown output weights $\boldsymbol{\beta}_{i}$ are determined by the batch Least Squares (LS) method. Thus, for the assigned matrix $\mathbf{H}[\cdot]$, suppose that its QR decomposition is a matrix of the form

$$
\mathbf{H}=\mathbf{Q R},
$$

where $\mathbf{R}$ is an upper triangular matrix and $\mathbf{Q}$ is an orthogonal matrix, i.e., the one satisfying

$$
\mathbf{Q}^{T} \mathbf{Q}=\mathbf{I}
$$

where $\mathbf{I}$ is the identity matrix. This matrix decomposition can be used to simplify the computation to determine the solution $\boldsymbol{\beta}$.

Assume that $\mathbf{R}$ is of full column rank. Then

$$
\hat{\boldsymbol{\beta}}=\left(\boldsymbol{R}^{T} \boldsymbol{R}\right)^{-1} \boldsymbol{R}^{T} \boldsymbol{Q}^{T} \boldsymbol{\Gamma}
$$

is the unique least-squares solution by minimizing

$$
J=\|\mathbf{H} \boldsymbol{\beta}-\mathbf{T}\|_{2}^{2} .
$$

\section{Experimental results}

Based on the experimental setup shown in Section 2, the experiment to measure the responses of acupoints was implemented. A sequence of semi-exponential signals, i.e., $x(t)=\beta\left(e^{-\alpha\left(t-t_{k}\right)}-1\right) 1\left(t-t_{k}\right)$, where $\beta=3.5$ and $\alpha=0.04$, was used to excite the acupoint Shaoshang (LU11) of the meridian system. The response of the acupoint Kongzui (LU6) was measured.

Firstly, we applied the standard ELM neural network to model hysteresis occurring in the meridian. Both the input and output nodes of the ELM network are set to one. The selection of the number of hidden neurons is shown in Table 1.

Note that the modelling performance of the standard ELM neural network is not improved even though the number of hidden neurons is increased. Among those cases, the neural model with 25 hidden neurons yielded the best validation solution.

In order to identify the hysteretic behaviour of the human meridian, the proposed method is utilized. The ELM neural model with self-feedback hidden neurons is constructed on the proposed expanded input space, i.e., the model has two inputs, i.e., one is the stimulating input and another is the output of the proposed hysteretic operator. For the structure of the dynamic ELM neural model, the selection procedure of the hidden neurons is presented in Table 2.

From Table 2, we see that when the number of hidden nodes is 15 , the best model validation result is obtained.

Table 1. Selection of the number of hidden neurons in the ELM model.

\begin{tabular}{|c|c|c|}
\hline $\begin{array}{c}\text { Number of } \\
\text { hidden nodes }\end{array}$ & $\begin{array}{c}\text { MSE of } \\
\text { training }\end{array}$ & $\begin{array}{c}\text { MSE of } \\
\text { validation }\end{array}$ \\
\hline \hline 10 & 0.1395 & 0.1418 \\
15 & 0.1346 & 0.1422 \\
20 & 0.1307 & 0.1402 \\
25 & 0.1308 & 0.1349 \\
30 & 0.1301 & 0.1398 \\
\hline
\end{tabular}




$$
\begin{array}{r}
\mathbf{H}(\mathbf{x}(t), f(\mathbf{x}(t)), z(\mathbf{x}(t-1), t-1)]=\left(\begin{array}{cc}
g\left(\mathbf{w}_{1} \cdot\left[x_{1}(t), f\left(\left(\mathbf{x}_{\mathbf{1}}(\mathbf{t})\right)\right)\right]\right)+\alpha_{1} z_{1}\left(\mathbf{x}_{1}(t-1), t-1\right) & \cdots \\
\vdots & \vdots \\
g\left(\mathbf{w}_{1} \cdot\left[x_{N}(t), f\left(\left(\mathbf{x}_{\mathbf{N}}(\mathbf{t})\right)\right)\right]\right)+\alpha_{1} z_{1}\left(\mathbf{x}_{N}(t-1), t-1\right) & \cdots \\
& g\left(\mathbf{w}_{1} \cdot\left[x_{N}(t), f\left(\left(\mathbf{x}_{\mathbf{N}}(\mathbf{t})\right)\right)\right]+\alpha_{1} z_{1}\left(\mathbf{x}_{N}(t-1), t-1\right)\right. \\
\vdots & \\
& g\left(\mathbf{w}_{\tilde{N}} \cdot\left[x_{N}(t), f\left(\left(\mathbf{x}_{\mathbf{N}}(\mathbf{t})\right)\right)\right]+\alpha_{\tilde{N}} z_{\tilde{N}}\left(\mathbf{x}_{N}(t-1), t-1\right)\right.
\end{array}\right),
\end{array}
$$
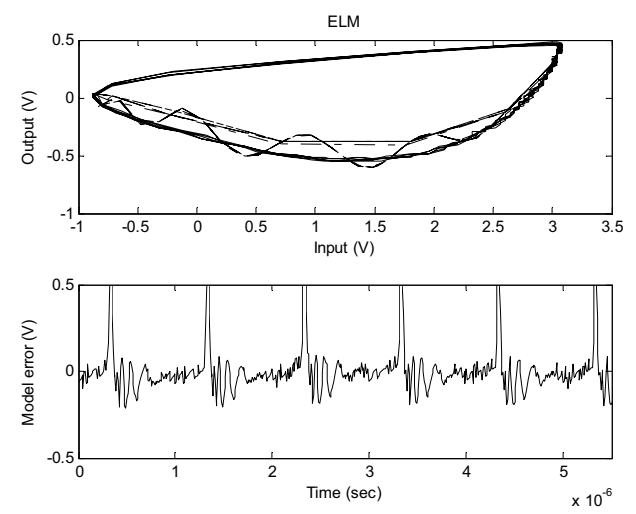

Fig. 5. Model validation of the standard ELM neural model.

Thus, we choose the architecture of the recurrent ELM neural network with two inputs, 15 hidden neurons and one output.

Figure 5 shows the model validation result of the ELM neural network based model and the corresponding modelling error. In Fig. 5, the solid line is the real data of the meridian and the dotted line is the obtained model data. The maximum modelling error is over $0.5 \mathrm{~V}$. In Fig. 5, it can be seen that the model failed to describe the hysteresis present in the meridian.

On the other hand, the proposed dynamic ELM neural network on the constructed expanded input space has obtained much better modelling performance even though the asymmetric hysteresis in the human meridian system is a rather complex behaviour. In the experiment, the train-

Table 2. Selection of the number of hidden neurons in the dynamic ELM model.

\begin{tabular}{|c|c|c|}
\hline $\begin{array}{c}\text { Number of } \\
\text { hidden nodes }\end{array}$ & $\begin{array}{c}\text { MSE of } \\
\text { training }\end{array}$ & $\begin{array}{c}\text { MSE of } \\
\text { validation }\end{array}$ \\
\hline \hline 10 & 0.0685 & 0.0800 \\
15 & 0.0234 & 0.0406 \\
20 & 0.0182 & 0.1282 \\
25 & 0.0152 & 0.2562 \\
30 & 0.0143 & 2.2458 \\
\hline
\end{tabular}
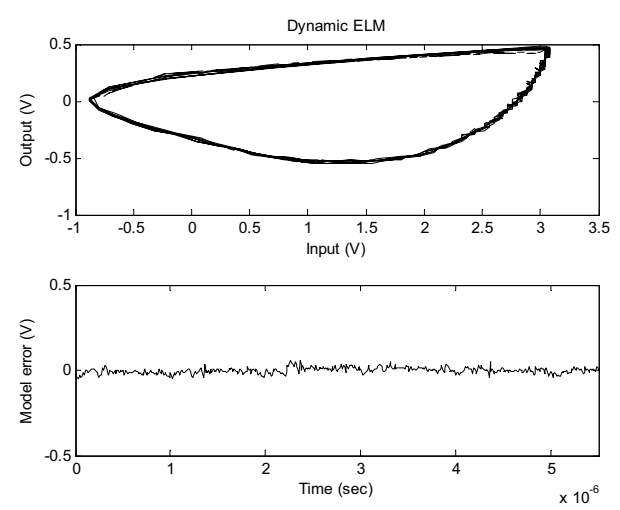

Fig. 6. Model validation of the proposed dynamic ELM neural model.

ing algorithm to specify the weights of the model was the same as that in the training of the ELM neural model. Figure 6 illustrates the corresponding performance of model validation. The dotted line is the modelling result while the solid line corresponds to the real data of the meridian system. Obviously, the modelling error of the proposed method is very small, just around zero. Moreover, the proposed method used a smaller number of hidden neurons than that of the conventional ELM neural model. Hence, the proposed modelling method has a much simpler model structure.

For modelling the response measured at the Lieque (LU7) point when Tianfu (LU3) is stimulated by a sawwave sequence with $10 \mathrm{KHz}$ in frequency and $4 \mathrm{~V}$ in peakto-peak amplitude. The proposed dynamic ELM neural model with 2 input nodes, 20 hidden nodes and 1 output is used to model this behavior. The corresponding model validation performance is shown in Fig. 7. It can be seen that satisfactory modeling performance is obtained.

For comparison, in this experiment, a Time-Delay Neural Network (TDNN) (Waibel, 1989) with local feedback is applied to the modeling of hysteresis in the meridian system. The architecture of the TDNN model consists of a two step time-delay in input nodes, 45 hidden nodes and one output node. The Levenberg-Marquardt 


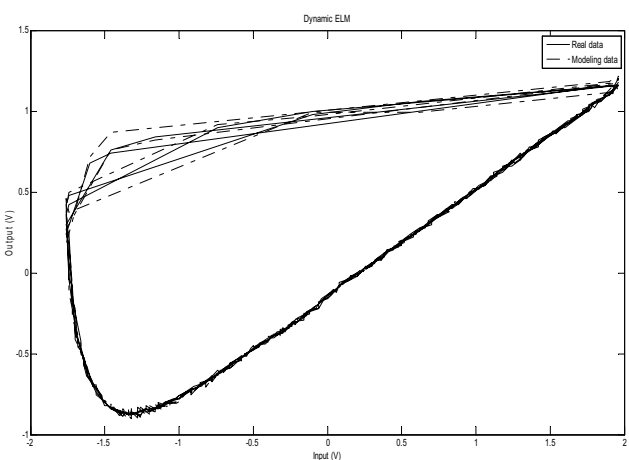

Fig. 7. Model validation of the proposed dynamic ELM method.

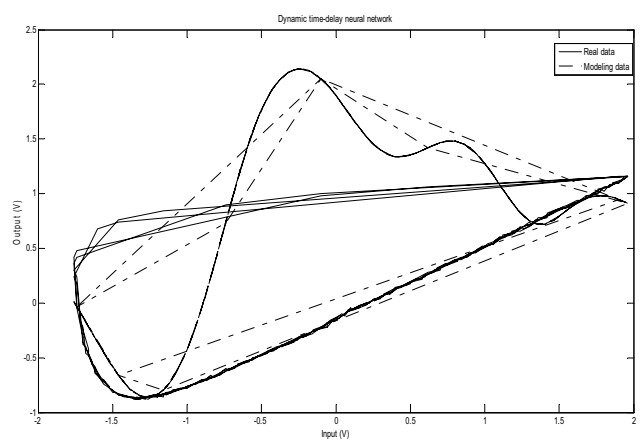

Fig. 8. Model validation of the time-delay neural network

(L-M) algorithm is implemented for model training. After 600 epochs, the corresponding model validation result is shown in Fig. 8. Obviously, it can be seen that a larger modeling error occurred. This is because of the multivalue effect of hysteresis and, probably, local minima.

\section{Conclusion}

In this paper, a modeling method for asymmetric hysteresis in human meridian systems is presented. In this modeling scheme, a modified hysteretic operator is proposed to construct an expanded input space to transform the multivalued mapping of hysteresis into a one-to-one mapping. On the constructed expanded input space, the ELM neural network is employed to model hysteresis inherent in human meridian systems. As hysteresis in the meridian system is an asymmetric and dynamic system, a dynamic ELM neural network is developed. In the proposed dynamic ELM neural network, the output state of each hidden neuron is fed back to its own input to describe the dynamic behavior of hysteresis. The training of the dynamic ELM neural network is rather simple. A least-squares algorithm based on QR decomposition is implemented.

Moreover, a TDNN modeling method is used to model the same hysteresis for comparison. However, the obtained TDNN model still fails to obtain a satisfactory modeling result.

The experimental results showed that the proposed dynamic ELM neural model based model obtained much better modeling performance and a simpler model structure.

\section{Acknowledgment}

This work was supported by the projects of Shanghai Normal University (DZL811, DRL904 and DYL201005), the projects of Shanghai Education Commission (11YZ92 and 13YZ056), the project of NSFC (Grants No. 61203108, 60971004 and 61171088), and the projects of the Science and Technology Commission of Shanghai (09220503000, 10JC1412200 and 09ZR1423400).

\section{References}

Ahn, C., Colbert, A.P., Anderson, B.J., Martinsen, O.G., Hammerschlag, R., Cina, S., Wayne, P.M and Langevin, H.M. (2008). Electrical properties of acupuncture points and meridians: A systematic review, Bioelectromagnetics 29(4): 245-256.

Dong, R. and Tan, Y. (2009). Modeling hysteresis in piezoceramic actuators using modified Prandtl-Ishlinskii model, Physica B 404(8-11): 1336-1342.

Ge, P. and Jouaneh, M. (1995). Modeling hysteresis in piezoceramic actuators, Precision Engineering 17(3): 211-221.

Huang, G. Zhu, Q. and Siew, C. (2006). Extreme learning machine: Theory and application, Neurocomputing 70: 489501.

Huang, G. and Chen, L. (2007). Convex incremental extreme learning machine, Neurocomputing 70(16-18): 30563062 .

Hu, H. and Mrad, R. (2003). On the classical Preisach model for hysteresis in piezoceramic actuators, Mechatronics 13(2): 85-94.

Macki, J.W., Nistri and Zecca, P. (1993). Mathematical models for hysteresis, SIMAC Review 35(1): 94-123.

Trentini, F.J., Thompson, B. and Erlichman, J.S. (2005). The antinociceptive effect of acupressure in rats, The American Journal of Chinese Medicine 33(1): 143-150.

Tsuei, J.J. (1998). A modern interpretation of acupuncture and the meridian system, 2nd International Conference on Bioelectromagnetism, Melbourn, Australia, pp. 177-182.

Wang, Z., Tan, Y. and Su, M. (2009). Modeling of meridian channels, Proceedings of the International Conference on Biomedical Electronics and Devices, Porto, Portugal, pp. 167-172.

Yang, H. (1997). The research and application of the dynamic testing system for point skin resistance, Journal of Biomedical Engineering 16(1): 41-50.

Yamamoto, Y. and Yamamoto, T. (1979). Dynamic system for the measurement of electrical skin impedance, Medical and Biological Engineering and Computing 17(1): 135137. 
Zhao, X. and Tan, Y. (2006). Neural network based identification of Preisach-type hysteresis in piezoelectric actuator using hysteretic operator, Sensors and Actuators A 126(2): 306311 .

Zhao, X. and Tan, Y. (2008). Modeling hysteresis and its inverse model using neural networks based on expanded input space method, IEEE Transactions on Control Systems Technology 16(3): 484-490.

Zhang, W., Xu, R. and Zhu, Z. (1999). The influence of acupuncture on the impedance measured by four electrodes on meridians, Acupuncture \& Electro-Therapeutics Research 24(3-4): 181-188.

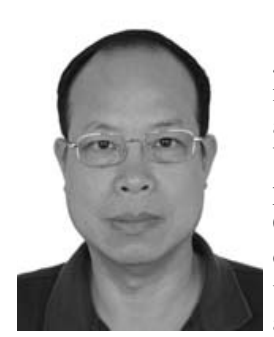

Yonghong Tan received B.Sc. and M.Sc. degrees from the South China University of Technology, Guangzhou, China. In 1996, he obtained a Ph.D. degree in electrical engineering from the University of Ghent, Belgium. Then, he was a post-doctoral fellow at Simon Fraser University, Canada. He has been a visiting professor at Colorado State University, USA, and Concordia University, Canada. He has also been a professor at the Guilin University of Electronic Technology and the University of Electronic Science and Technology of China. Presently, he is a full professor at the College of Mechanical and Electronic Engineering, Shanghai Normal University, China. His research interests are modeling and control of nonlinear systems, biomedical signal processing and intelligent control.

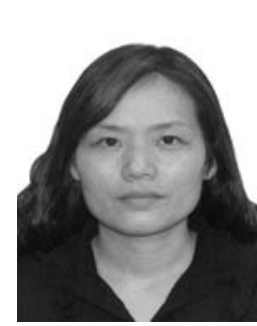

Ruili Dong received both a B.Sc. degree in mechanical engineering and an M.Sc. degree in electrical engineering from Northwestern Polytechnical University, Xi' an, China. In 2009, she obtained a Ph.D. degree from the Department of Automation, Shanghai Jiaotong University, Shanghai, China. Presently, she is an associate professor at the College of Mechanical and Electronic Engineering, Shanghai Normal University. Her research interest is modeling and control for nonlinear dynamic systems.

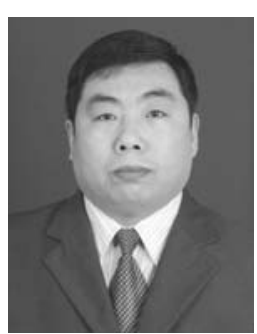

Hui Chen is now a Ph.D. candidate at the School of Automation, Southeast University, Nan-jing, China. His current research interests include modeling and control of nonlinear systems, and intelligent control.

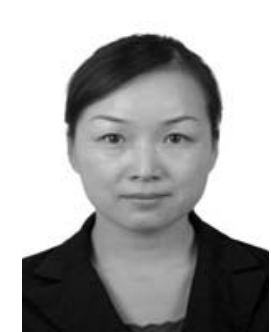

Hong He obtained her Ph.D at the East China University of Science and Technology, China, in 2008. Now she is an associate professor at Shanghai Normal University. Her research direction includes computational intelligence theory and its application in system modeling, control and pattern recognition.

Received: 11 March 2011

Revised: 24 November 2011

Re-revised: 12 May 2012 